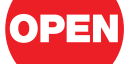

SUBJECT AREAS:

COMPUTATIONAL

BIOLOGY

DISEASES

BIOLOGICAL MODELS

ANIMALS

Received

10 November 2011

Accepted

27 February 2012

Published

19 March 2012

Correspondence and requests for materials should be addressed to J.T. (j.turner@liverpool. ac.uk)

\section{Modelling bluetongue virus transmission between farms using animal and vector} \section{movements}

\author{
Joanne Turner', Roger G. Bowers² \& Matthew Baylis'
}

\author{
'Department of Epidemiology and Population Health, Institute of Infection and Global Health, University of Liverpool, UK, \\ ${ }^{2}$ Department of Mathematical Sciences, School of Physical Sciences, University of Liverpool, UK.
}

Bluetongue is a notifiable disease of ruminants which, in 2007, occurred for the first time in England. We present the first model for bluetongue that explicitly incorporates farm to farm movements of the two main hosts, as well as vector dispersal. The model also includes a seasonal vector to host ratio and dynamic restriction zones that evolve as infection is detected. Batch movements of sheep were included by modelling degree of mixing at markets. We investigate the transmission of bluetongue virus between farms in eastern England (the focus of the outbreak). Results indicate that most parameters affecting outbreak size relate to vectors and that the infection generally cannot be maintained without between-herd vector transmission. Movement restrictions are effective at reducing outbreak size, and a targeted approach would be as effective as a total movement ban. The model framework is flexible and can be adapted to other vector-borne diseases of livestock.

luetongue is a notifiable disease that affects all ruminants. Sheep are often severely affected by the disease while cattle are considered to be the main reservoir host. The infection is endemic in warm parts of the world, including Africa ${ }^{1}$, south Asia, Australasia and the Americas. In Europe, the infection has occurred in the form of periodic epidemics in the southern Mediterranean region ${ }^{2,3}$ over several decades. In recent years this pattern has altered, possibly in response to climate change $e^{4}$, and the first outbreak of bluetongue in Northern Europe occurred in 2006. The following year, the disease spread to the UK. Although movement restrictions limited the spread of the disease within the UK, confirmed cases (indicated by unique Bluetongue Disease (BTD) numbers) had been recorded across six counties in the south east of the country by the end of October 2007 (Figure 1a). By $18^{\text {th }}$ December 2008, there were 151 confirmed cases (including 12 identified by post-import testing) (Figure 1b). A large number of these were located in Norfolk and Suffolk, the likely point of introduction ${ }^{5}$.

Various models have been developed to explore the northern European and UK outbreaks ${ }^{6-8}$. In some cases, only a single mode of transmission is considered. For example, Hendrickx et al. ${ }^{7}$ considers vector transmission only. In other cases, transmission between farms is modeled using a single transmission kernel ${ }^{8}$, which includes all modes of transmission but does not allow different transmission mechanisms to be investigated. Here, we present the first model for bluetongue that explicitly incorporates farm to farm movements of the two main hosts, as well as vector dispersal. Realistic farm to farm contacts were incorporated by using recorded animal movements (from the Animal Movement Licensing System (AMLS) and the Cattle Tracing System (CTS) for 2006). The model was further enhanced by including a seasonal vector to host ratio based on the formula given in Lord et al. ${ }^{9}$ (developed for the Afro-asiatic vector) with parameter values modified to suit the UK season. A recent paper ${ }^{10}$ examines seasonal patterns of abundance of UK vector species. The start dates and lengths of the vector periods given in Figure 3 of Sanders et al. ${ }^{10}$ are consistent with the values used here. However, as the authors did not trap on or near livestock, we cannot assume that the sizes of the catches approximate vector to host ratios. The data would have to be validated against catches on animals before they could be used in epidemiological models. Dynamic restriction zones (based on the movements table in Defra's UK Bluetongue Control Strategy published in August $2007^{11}$ ) that evolve as infection is detected were also incorporated into the model. Batch movements of sheep were included by introducing a parameter that governs the degree of mixing at markets, and hence affects the number of suppliers contributing to each batch of sheep.

The probability that a farm becomes exposed or infected as a result of buying in animals takes into account the number of animals received from each supplier and the proportion of exposed or infected animals on each source farm. The model incorporates the mandatory 6-day movement standstill for any farm that receives cattle or sheep. 
(a)

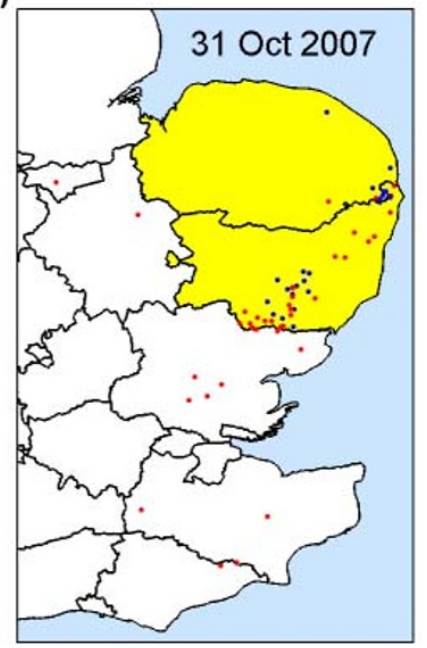

(b)

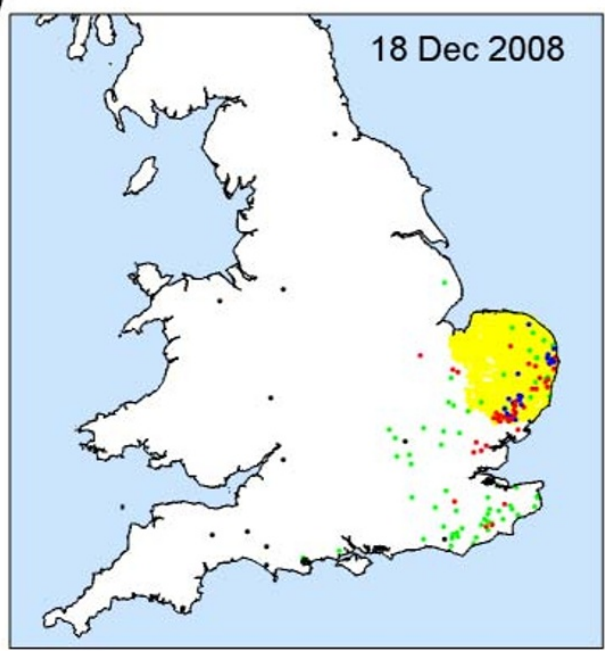

Figure 1 Spatial distribution of confirmed cases. It has been estimated that bluetongue infected midges were blown into the UK on 5 th August $2007^{5}$. By 31st October 2007, 61 cases had been confirmed (Figure 1a). By 18th December 2008, the number had risen to 151 (Figure 1b). Confirmed cases are coloured according to method of detection. Confirmed cases identified by post-import test notification are not considered to be indigenous confirmed cases of BTV. The counties of Suffolk and Norfolk (i.e. the area covered by our model) are shown in yellow. Key: Red = Reported case; Blue = Identified through surveillance, surveillance plus pre-movement testing or overwintering survey; Green = Identified through pre-movement testing, private testing or tracing; Black $=$ Post-import test notification.

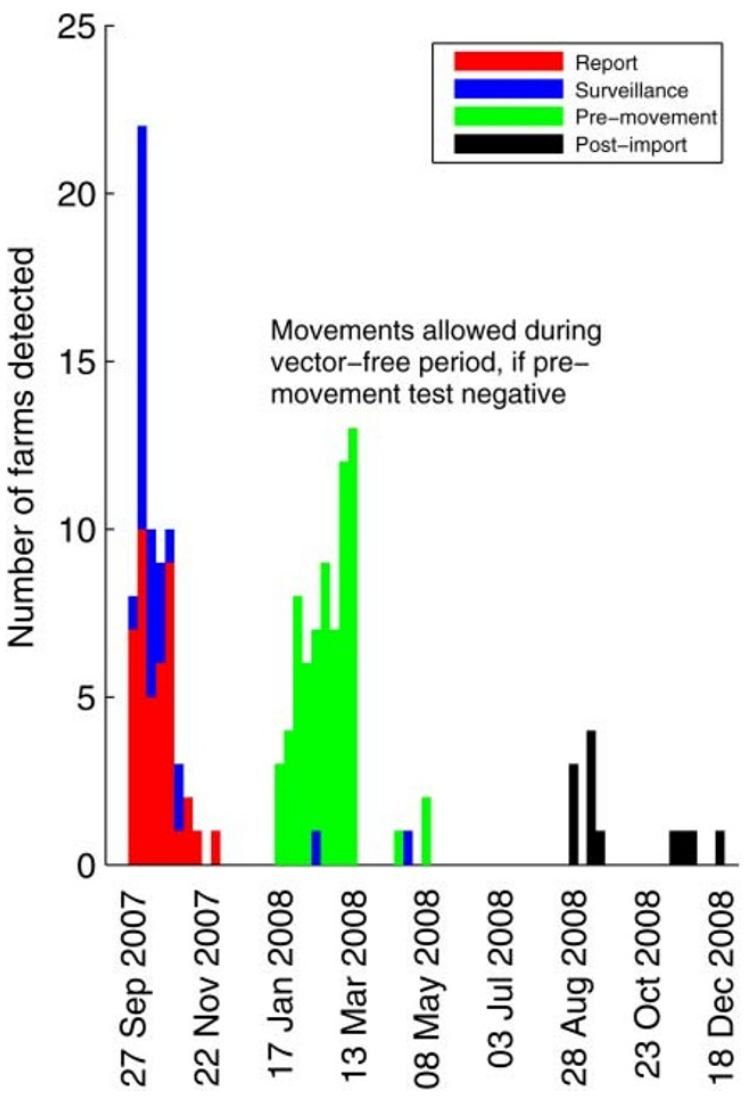

Figure $2 \mid$ Stacked bar chart showing the number of confirmed cases recorded per week. Colour indicates method of detection. Key: Red = Reported case; Blue = Identified through surveillance, surveillance plus pre-movement testing or overwintering survey; Green = Identified through pre-movement testing, private testing or tracing; Black $=$ Postimport test notification. (a)

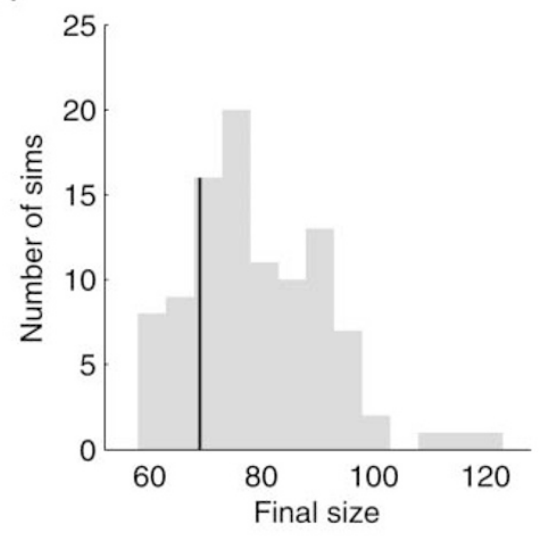

(b)

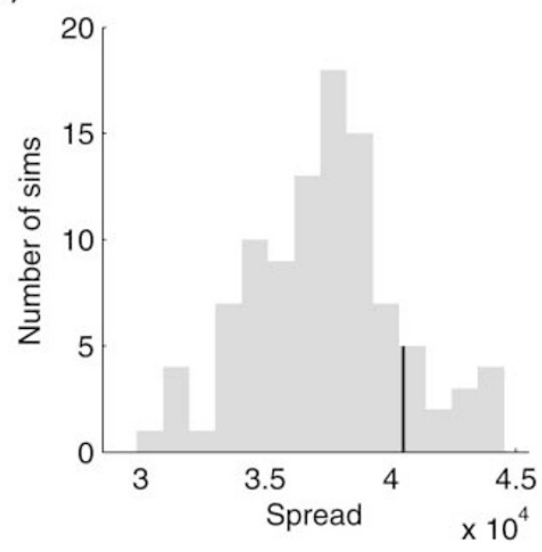

Figure $3 \mid$ Distributions of (a) final size and (b) spread (in metres) for the model with two index cases and infection introduced on 5th August. The vertical lines mark the positions of the observed values for Suffolk and Norfolk, which were 69 and $4.05 \times 10^{4} \mathrm{~m}$ for final size and spread, respectively. 
Average monthly temperatures were estimated for each location using Met Office data for $2000-2005(5 \mathrm{~km} \times 5 \mathrm{~km}$ resolution). Temperature was used to switch between periods of vector activity $\left(>10.5^{\circ} \mathrm{C}\right)$ and periods when the vector was assumed to be inactive $\left(\leq 10.5^{\circ} \mathrm{C}\right)$. Temperature affects the probability of exposure through vector movement (i.e. the probability that a farm becomes exposed as a result of animals being bitten by infectious vectors from a neighbouring infectious farm) and on-farm prevalence (estimated using an epidemic curve suggested by Pongsumpun et al. ${ }^{12}$ ). Further details of the model are given in the Supplementary Material.

As a large number of confirmed cases were located in Suffolk and Norfolk, we initially focus on transmission within these two counties. UK-wide transmission and the effects of climate change will be considered in a later publication. Daily temperature data will be used in this later study. Its greatest impact is likely to be on calculations at the beginning and end of the vector period (i.e. where temperatures are close to the cut-off of $10.5^{\circ} \mathrm{C}$ ). This level of detail will be important when comparing potential outbreaks in the north and south of the country, but is unwarranted within the smaller area of Suffolk and Norfolk.

Models are essential tools needed to explore control options, and this model is designed to allow various options to be investigated. As the model includes transmission via two different routes (animal and vector), a seasonal vector to host ratio and dynamic restriction zones, it differs considerably from other bluetongue models. Szmaragd et al. ${ }^{8}$ highlight the need for models that incorporate, among other things, separate transmission routes and seasonal vector dynamics. In incorporating these factors, this model represents a substantial improvement on existing models.

\section{Results}

Modelling the 2007 UK outbreak data. Data on the UK 2007-2008 bluetongue outbreak was supplied by Defra's National Emergency Epidemiology Group (NEEG). Figure 1 shows the spatial distribution of confirmed cases with colour indicating the method of detection. Figure 2 shows the number of confirmed cases recorded per week from 27 September 2007 - 18 December 2008. In the initial phase of the outbreak (Sep - Dec 2007), 66 confirmed cases were recorded: 42 were reported cases; 24 were identified through surveillance testing. From Jan - Mar 2008 (i.e. during the vector-free period), a further 69 cases were confirmed: 65 as a result of pre-movement testing. It is likely that the cases detected during this period were infected during the initial phase of the outbreak. This makes it difficult to estimate the rate of transmission. The problem is further compounded by the fact that there was an outbreak of FMD in Surrey at the same time, prompting a national livestock movement ban. Consequently, there were two sets of movement restrictions in place. As our model imposes bluetongue movement restrictions only, we cannot hope to match exactly the pattern of spread observed in 2007. However, the general picture should be the same.

There is evidence to suggest that infection arrived in the UK on the night of the $4^{\text {th }} / 5^{\text {th }}$ August $2007^{5}$ and Figure 1 in Defra's initial epidemiological report into the UK outbreak ${ }^{13}$ suggests that it was introduced into two distinct areas of Suffolk and Norfolk. So, we first ran our model with two index cases, namely the first two animal holdings identified as infected. Using the point estimates given in Table S3, we ran the model 100 times to capture stochastic variation in model output. Figure 3 shows the distributions of final size and spread (average distance between new cases and first index case) obtained by the end of a year in which infection was introduced on $5^{\text {th }}$ August. The vertical lines mark the positions of the observed values for Suffolk and Norfolk. These values include reported cases and those detected by surveillance and pre-movement testing before 15 March 2008, and are consistent with the model output.

Figure 4 shows spatial plots corresponding to one of the realisations contributing to Figure 3. It illustrates that the model includes both local and more long-range transmission, and demonstrates the dynamic nature of the restriction zones imposed following detection. (a)

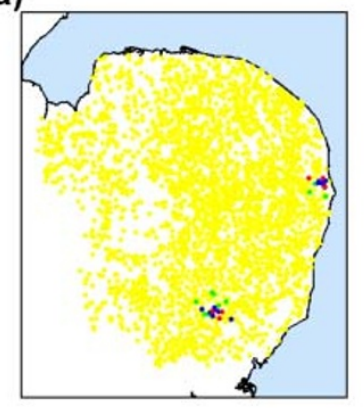

(d)

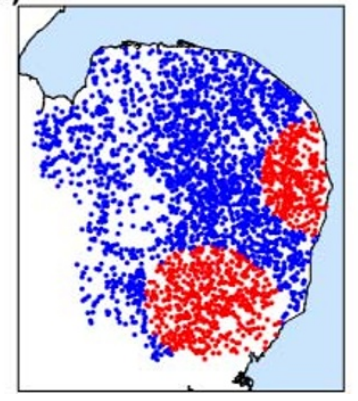

(b)

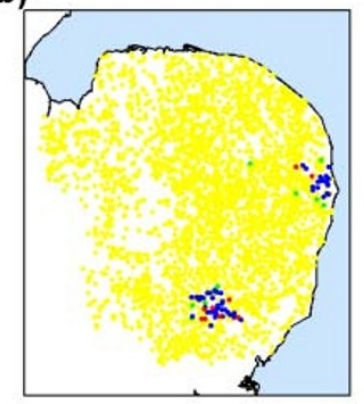

(e)

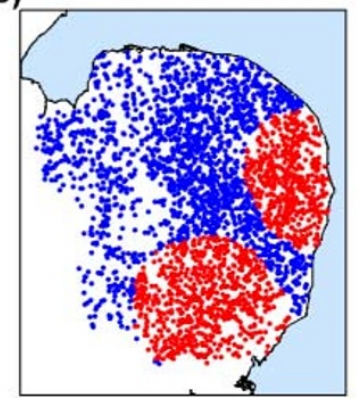

(c)

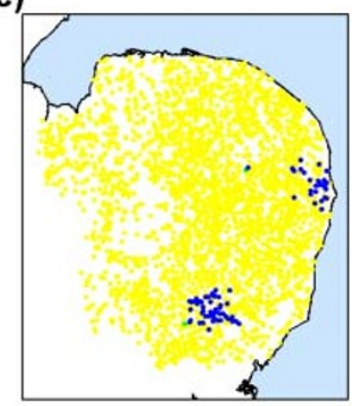

(f)

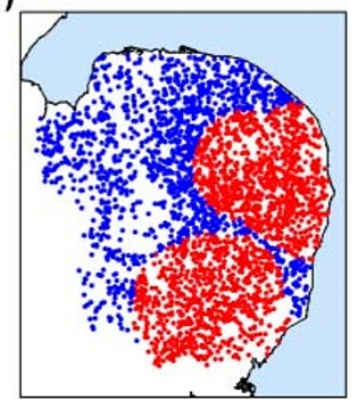

Figure $4 \mid$ One realisation of the model generated using the point estimates given in Table S3. The figure shows the status of farms in Suffolk and Norfolk at the end of (a) August, (b) September and (c) October after starting with two infectious farms on the 5th August. Colour indicates infection status: Yellow = 'susceptible' (S); Green = 'exposed' (E or W $\mathrm{SE}_{\mathrm{SE}}$; Red = 'infectious' ( $\mathrm{I}, \mathrm{W}_{\mathrm{EI}}$ or $\left.\mathrm{W}_{\mathrm{SI}}\right)$; Blue = 'detected' (D). The corresponding plots (d), (e) and (f) show the extent of the restriction zones at the same times. Colour indicates zone type: Yellow = 'disease-free area'; Red = 'control zone' (CZ); Blue $=$ 'protection zone' $(\mathrm{PZ})$; Green $=$ 'surveillance zone' $(\mathrm{SZ})$. 
In Figure $4 \mathrm{~b}$, the distance from the upper cluster to the isolated exposed farm is almost $20 \mathrm{~km}$ : too far for vector transmission. Therefore, this exposure must be the result of within-zone animal movement (which is allowed). Figure 4 also reveals that our detection mechanism, which relies purely on detection of clinical signs, is too efficient. In reality, less than half of the farms infected had been detected by the end of December 2007 as a result of animals displaying clinical signs (Figure 2). In our example, almost all of those infected by the end of October have already been detected. Although our faster rate of detection leads to movement restrictions being imposed and adjusted more quickly in our model, the area covered by our control zone at the end of October is only about half the area of Suffolk and Norfolk. Defra's initial control zone (imposed on 29 September 2007) covered almost all of Suffolk and Norfolk,

(a)

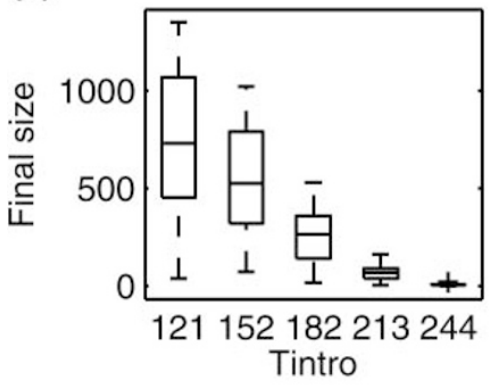

(c)

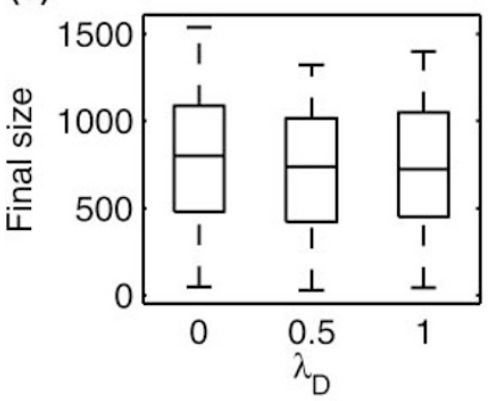

(e)

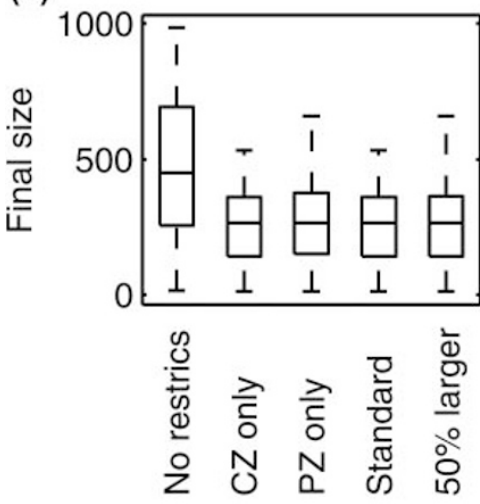

indicating that it was not based entirely on confirmed cases. Defra's UK Bluetongue Control Strategy ${ }^{11}$ explains that their restriction zones take into account natural boundaries to the dispersal of vectors as well as administrative and other factors.

Testing scenarios using the model. The results for each scenario are based on 100 simulations. Each simulation begins with a single, randomly-chosen index case. The results are shown in Figure 5, which contains distributions of final size for different parameter values and movement settings. As sheep are generally more severely affected and therefore infection is likely to be detected more quickly on farms containing sheep, farms were classified as either cattle only, sheep only or mixed. The first two figures relate to the time of introduction of infection (Tintro, 5a) and the farm type

(b)

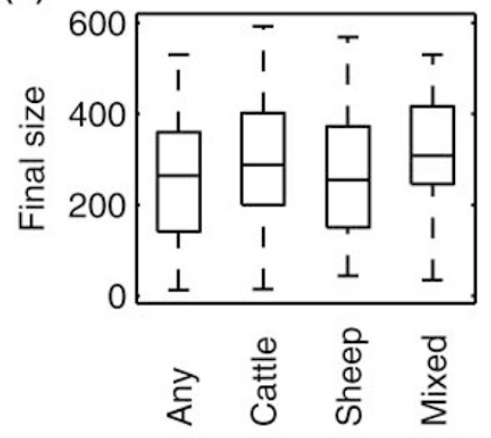

(d)

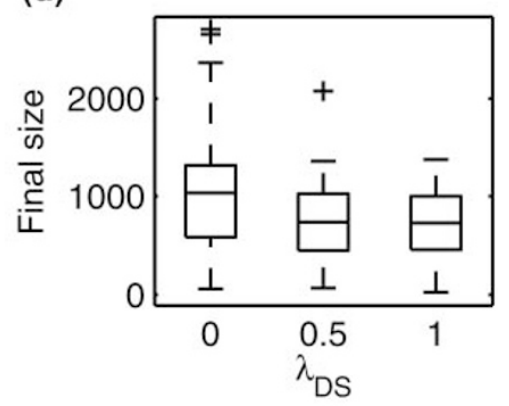

(f)

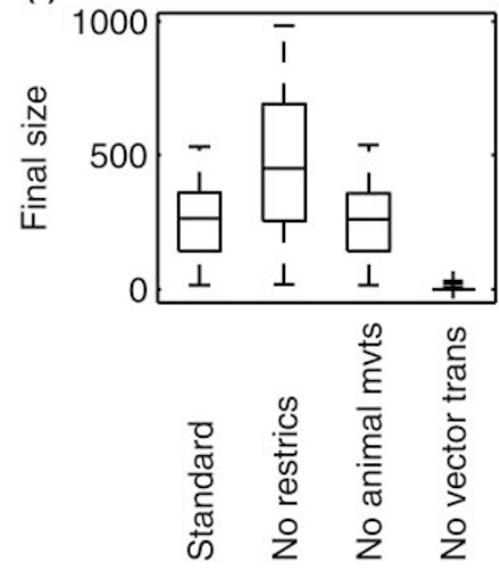

Figure 5 Results for the bluetongue simulation model obtained using the parameter estimates given in Table S3 unless otherwise stated. Each distribution is based on 100 simulations: (a) different start times (Tintro $=$ time infection introduced), (b) different farm types (Fintro $=$ farm type of index case), (c) different $\lambda_{D}$ values $\left(\lambda_{D}\right.$ indicates proportion of infected cattle showing clinical signs, Tintro $\left.=121\right)$, (d) different $\lambda_{D S}$ values $\left(\lambda_{D S}\right.$ indicates proportion of infected sheep showing clinical signs, Tintro $=121)$, (e) different zone sizes and configurations (as described in the main text) and (f) different movement settings (as described in the main text). 
(a)

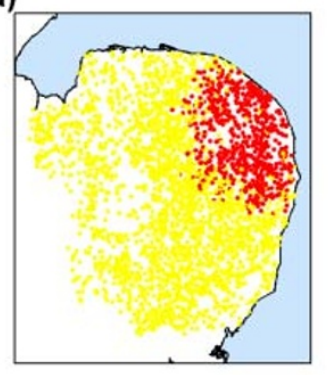

(b)

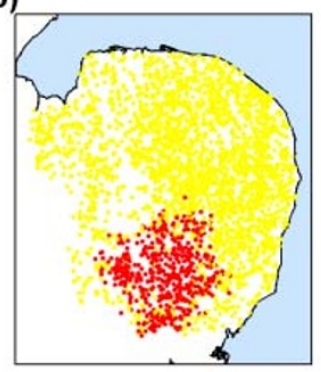

(c)

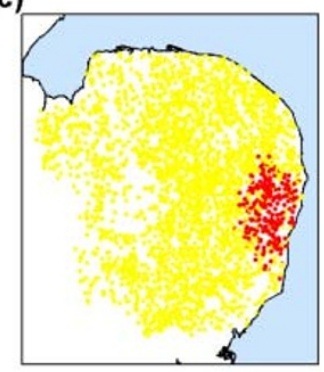

(d)

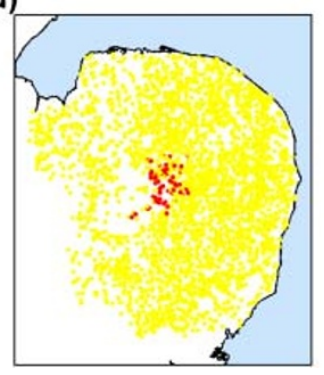

(e)

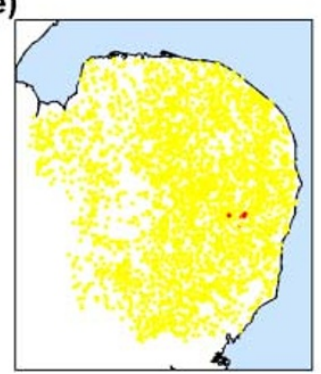

Figure 6 Spatial plots corresponding to Figure 5a. (a) Tintro $=121$, median $=731.5$, example final size $=731$, (b) Tintro $=152$, median $=525.5$, example final size $=524$, (c) Tintro $=182$, median $=264.5$, example final size $=265$, (d) Tintro $=213$, median $=68.5$, example final size $=66$, (e) Tintro $=244$, median $=5$, example final size $=5$.

of the index case (Fintro, 5b) - both of which are factors that cannot be controlled (except within the model). The next two figures relate to the proportions of infectious cattle (5c) and sheep (5d) that show clinical signs and hence to the rates of detection - both of which are largely unknown. The last two figures $(5 e, 5 f)$ consider different movement restrictions and scenarios and so relate to possible intervention measures.

Figure $5 \mathrm{a}$ reveals the effect of time of introduction of infection. Five different start times are considered, namely $1^{\text {st }}$ May (Tintro $\left.=121\right), 1^{\text {st }}$ June $($ Tintro $=152), 1^{\text {st }}$ July $($ Tintro $=182), 1^{\text {st }}$ August $($ Tintro $=218)$ and $1^{\text {st }}$ September (Tintro $=244$ ). As one might expect, the earlier the infection is introduced, the larger the outbreak. The specific relationship is likely to be affected by the average ratio of vectors to hosts, which is seasonal and peaks in July. Figure $5 b$ reveals that the farm type of the index case has no significant effect on final size.

Figures $5 \mathrm{c}$ and $5 \mathrm{~d}$ show how final size is affected by the proportions of infectious cattle $\left(\lambda_{D}\right)$ and sheep $\left(\lambda_{D S}\right)$ that show clinical signs. In Figure $5 c, \lambda_{D S}$ is close to 1 while $\lambda_{D}$ varies from 0 to 1 . Consequently, we see only a small reduction in final size as $\lambda_{D}$ increases. In Figure $5 \mathrm{~d}, \lambda_{D}$ is close to zero while $\lambda_{D S}$ varies from 0 to 1 . As a result, we see a larger reduction in final size as $\lambda_{D S}$ increases. However, the effect is not significant.

Figure $5 \mathrm{e}$ compares the effects of the following movement restrictions (see Table S3 for parameter definitions):

- "No restrictions": no movement restrictions are imposed (achieved with $\lambda_{D}=\lambda_{D S}=0$ );

- "CZ only": a single $20 \mathrm{~km}$ control zone is imposed around each detected farm $(\mathrm{CZrad} 1=\mathrm{PZrad} 1=$ SZrad $1=20)$;

- "PZ only": a single $100 \mathrm{~km}$ protection zone is imposed around each detected farm $(\mathrm{CZrad} 1=\mathrm{PZrad} 1=\operatorname{SZrad} 1=100)$;

- "Standard": standard animal movement restrictions are imposed $(\mathrm{CZrad} 1=20, \mathrm{PZrad} 1=100, \mathrm{SZrad} 1=150)$;

- “50\% larger": standard (i.e. three zone) approach is adopted, but all radii are 50\% larger $(\mathrm{CZrad} 1=30, \mathrm{PZrad} 1=150$, SZrad1 $=225)$.

This figure shows that animal movement restrictions are effective in reducing final size, but also suggests that imposing a single $20 \mathrm{~km}$ restriction zone around each detected farm is just as effective as imposing more or larger zones. However, it is possible that larger zones would prove more effective if the model incorporated windassisted (and hence longer-distance) dispersal of vectors.

Figure $5 \mathrm{f}$ compares the effects of the following movement scenarios:

- "Standard": includes animal and vector movements, and standard animal movement restrictions;

- "No restrictions": no movement restrictions are imposed (achieved with $\lambda_{D}=\lambda_{D S}=0$ );

- "No animal mvts": no animals are moved between farms;

- "No vector trans": no between-farm vector to host transmission (achieved with $\beta_{v h}=0$ ) and no movement restrictions (achieved with $\left.\lambda_{D}=\lambda_{D S}=0\right)$.

It suggests that a targeted ban on animal movements ("Standard") is effective in reducing the size of an outbreak (when compared with "No restrictions") and that a targeted ban is just as effective as a total ban ("No animal mvts"). It also reveals that while animal movements contribute to transmission, the infection generally cannot be maintained without between-herd vector transmission.

Figure 6 contains spatial plots corresponding to Figure 5a. Each plot shows the farms infected during a simulated outbreak with a size approximately equal to the median of the corresponding distribution. As the index case is chosen at random in each simulation, the plots also show how the disease spreads in different areas of the region (e.g. where farm density is low). Similarly, Figure 7 contains spatial plots corresponding to Figure $5 f$. These clearly demonstrate the long-range spread possible when no animal movement restrictions are imposed and that the standard targeted ban is just as effective in reducing that spread as a total ban on animal movements.

We also considered the effect that $q$ (degree of mixing at market) might have on the transmission of bluetongue. Results (not shown) indicate that it has virtually no effect on final size. A slight difference can be seen when movement restrictions are not imposed. This difference is more pronounced when the number of animal holdings involved is large. However, the AMLS data indicate that in 2006 this number was just 361 (i.e. less than $11 \%$ of all animal holdings). After verifying that indirect sheep movements are not more long-range 
(a)

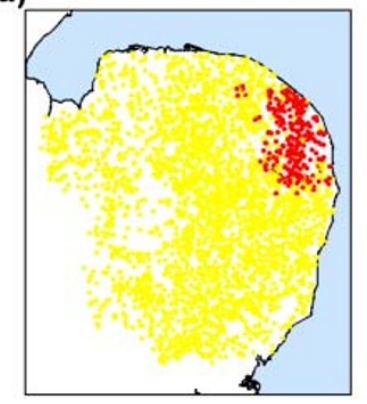

(b)

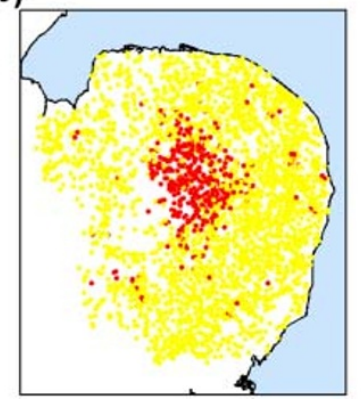

(c)

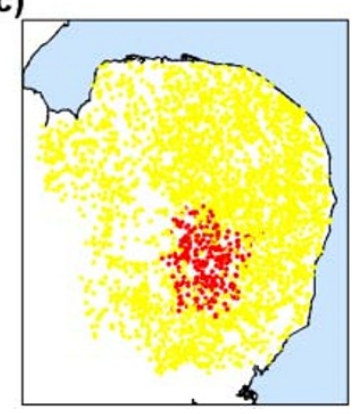

Figure $7 \mid$ Spatial plots corresponding to Figure 5f. (a) Standard movement restrictions, median $=264.5$, example final size $=265$, (b) No movement restrictions, median $=450$, example final size $=446$, (c) No animal movements, median $=260.5$, example final size $=264$.

than direct sheep movements, we concluded that the lack of an effect on final size was due to the fact that movement restrictions reduced the already small number of indirect sheep movements to a level that was insignificant in comparison to the large number of cattle and direct sheep movements.

Sensitivity analysis. After investigating the effects of Tintro, Fintro, $q$ and various movement scenarios, a sensitivity analysis was performed on the remaining parameters. Some were found to have a significant effect on the final size and spread of an outbreak (Table S4). The majority of these related to vector transmission. However, three were connected to the prevalence curve, which approximates the on-farm dynamics during the vector period. Another was related to how quickly a farm becomes 'infectious' after becoming 'exposed'.

\section{Discussion}

This model allows us to focus on underlying mechanisms of transmission, in particular the roles of animal and vector movement. It also allows us to assess the impact of factors that in reality cannot be controlled (e.g. time of introduction of infection and farm type of index case), factors with high uncertainty (e.g. proportion of infectious animals that show clinical signs) and those related to possible intervention measures (i.e. things that can be controlled such as movement restrictions). After looking in detail at certain specific parameters, the effects of the remaining parameters were assessed by comparing partial rank correlation coefficients.

The results of the sensitivity analysis indicate that the majority of parameters affecting the final size of an outbreak relate to vector transmission. This is supported by Figure 5f, which shows that the infection generally cannot be maintained without between-herd vector transmission. This emphasises the need for further work on native vectors of bluetongue. More accurate information is required on how far they can travel in a day, their susceptibility to infection and ability to transmit the virus, the extrinsic incubation period and their mortality and biting rates. However, three of the parameters affecting final size are connected to the prevalence curve, which approximates the on-farm dynamics during the vector period. Another is related to how quickly a farm becomes 'infectious' after becoming 'exposed'. Exploration of a within-farm model is needed in order to improve these parameter estimates.

The results relating to animal movements and movement restrictions are very interesting, suggesting that movement restrictions are effective at reducing the size of an outbreak, but that a targeted approach (even just a single $20 \mathrm{~km}$ restriction zone around each infected holding) would be just as effective as a total movement ban. However, it is possible that a model incorporating wind-assisted spread of vectors might favour larger restriction zones (see comment below). The results relating to the proportions of infectious cattle and sheep that show clinical signs suggest that there is little point in trying to improve these estimates. Although they are related to the rates at which farms are detected (and movement restrictions are laid down), they seem to have little effect on final size. Similarly, the degree of mixing at market has virtually no effect on final size. Our investigations indicate that markets would not be an appropriate target for intervention, unless there was an increase in the number of holdings trading batches of sheep via this route.

It is widely accepted that wind can assist vectors to disperse great distances over large bodies of water. However, the sort of long-range $(>100 \mathrm{~km})$ spread seen over sea does not occur over land. Hendrickx et al. ${ }^{7}$ show that $50 \%$ of cases occur within $5 \mathrm{~km}$ of the previous case, while $95 \%$ of cases occur within $31 \mathrm{~km}$ of it. They also point out that vectors can fly short-distances both upwind and downwind at low to zero wind-speeds. For this reason, we chose not to include a wind component in this model. However, longer-distance dispersal downwind at high wind-speeds can occur, and this could have a marked effect on the spatial pattern of spread and, as a result, implications for the shape and size of restriction zones.

The model developed here has temperature-sensitive components and can be used to explore the effect of climate variability and climate change on the transmission, spread and persistence of bluetongue subsequent to the introduction of BT virus; it therefore complements a recent model of the effects of climate variability and change on the risk of bluetongue outbreaks occurring after the initial introduction of BT virus into fully susceptible host populations ${ }^{4}$. An exploration of the effects of climate will be the focus of a later publication but, for now, there are two points worth noting. From the formula for the seasonal vector to host ratio $m(t)$ (Table S3), we can conclude that an increase in the amplitude $T_{A}$ of the annual temperature fluctuation would produce the same effect as an increase in $\tilde{\rho}$, which leads to a higher ratio of vectors to hosts during the vector period and a consequent increase in final size and spread. Secondly, the sensitivity analysis reveals that the final size and spread of an outbreak are highly sensitive to parameters governing the seasonally-varying ratio of vectors to hosts and the temperature-dependent biting, extrinsic incubation and vector mortality rates. Therefore, any investigation into the effects of climate change should also take into account changes and uncertainty in these parameters.

\section{Methods}

Monte Carlo simulation. Parameter values (either point estimates, random samples from parameter distributions or specific values associated with interventions) were grouped to form parameter sets. The model was run 100 times for each parameter set in order to capture stochastic variability. Results are presented in the form of distributions of final size and spread. Spatial plots from individual simulations are also produced.

Sensitivity analysis. First 100 parameter sets were created by selecting parameter values uniformly from the ranges given in Table S3, then running 100 simulations for each parameter set and taking the median final size and spread. Partial rank correlation coefficients (PRCC's) were then calculated as described in Blower and Dowlatabadi ${ }^{14}$. The results are presented in Table S4: the larger the value, the greater the effect. A minus sign denotes a negative correlation. PRCC values with a magnitude greater than 0.3017 (the $1 \%$ critical value for results based on 100 parameter sets) are 
considered to be significant and shown in bold.

1. Paweska, J. T., Venter, G. J. \& Mellor, P. S. Vector competence of South African Culicoides species for bluetongue virus serotype 1 (BTV-1) with special reference to the effect of temperature on the rate of virus replication in C. imicola and C. bolitinos. Med. Vet. Entomol. 16, 10-21 (2002).

2. Mellor, P. S. \& Wittmann, E. J. Bluetongue virus in the Mediterranean basin 19982001. Vet. J. 164, 20-37 (2002).

3. Purse, B. V. et al. Climate change and the recent emergence of bluetongue in Europe. Nat. Rev. Microbiol. 3, 171-181 (2005).

4. Guis, H. et al. Modelling the effects of past and future climate on the risk of bluetongue emergence in Europe. J. R. Soc. Interface (Published online, 2011). (DOI:10.1098/rsif.2011.0255)

5. Gloster, J., Burgin, L., Witham, C., Athanassiadou, M. \& Mellor, P. S. Bluetongue in the United Kingdom and northern Europe in 2007 and key issues for 2008. Vet. Rec. 162, 298-302 (2008)

6. Gerbier, G. et al. Modelling local dispersal of bluetongue virus serotype 8 using random walk. Prev. Vet. Med. 87, 119-130 (2008).

7. Hendrickx, G. et al. A wind density model to quantify the airborne spread of Culicoides species during north-western Europe bluetongue epidemic, 2006. Prev. Vet. Med. 87, 162-181 (2008).

8. Szmaragd, C. et al. A modelling framework to describe the transmission of bluetongue virus within and between farms in Great Britain. PLoS ONE 4, e7741 (2009).

9. Lord, C. C., Woolhouse, M. E. J., Rawlings, P. \& Mellor, P. S. Simulation studies of African horse sickness and Culicoides imicola (Diptera: Ceratopogonidae). J. Med. Entomol. 33, 328-338 (1996).

10. Sanders, C. J. et al. Influence of season and meteorological parameters on flight activity of Culicoides biting midges. J. Appl. Ecol. 48, 1355-1364 (2011)

11. UK Bluetongue Control Strategy (Defra, August 2007)

12. Pongsumpun, P. et al. Dynamics of dengue epidemics in urban contexts. Trop. Med. Int. Health 13, 1180-1187 (2008).

13. National Emergency Epidemiology Group. Initial Epidemiological Report On The Outbreak Of Bluetongue In East Anglia And South East England From Investigations Completed To 19 October 2007 (Defra, 2007).
14. Blower, S. M. \& Dowlatabadi, H. Sensitivity and uncertainty analysis of complex models of disease transmission: an HIV model, as an example. Int. Stat. Rev. 62, 229-243 (1994).

\section{Acknowledgements}

This work was funded by The Leverhulme Trust Research Leadership Award F/0025/AC "Predicting the effects of climate change on infectious diseases of animals" awarded to Matthew Baylis. The authors would also like to thank the following people for their contribution: National Emergency Epidemiology Group (NEEG) for supplying CTS, AMLS, Agricultural Survey and bluetongue case data; Christian Setzkorn for extracting relevant information from the CTS data; Helene Guis for supplying average monthly temperatures for each holding; Rob Christley and Jonathan Read for initial discussions about the model.

\section{Author contributions}

All three authors contributed to the development of the model and main manuscript text. JT analysed the model and prepared the figures.

\section{Additional information}

Supplementary information accompanies this paper at http://www.nature.com/ scientificreports

Competing financial interests: The authors declare no competing financial interests.

License: This work is licensed under a Creative Commons

Attribution-NonCommercial-ShareAlike 3.0 Unported License. To view a copy of this license, visit http://creativecommons.org/licenses/by-nc-sa/3.0/

How to cite this article: Turner, J., Bowers, R.G. \& Baylis, M. Modelling bluetongue virus transmission between farms using animal and vector movements. Sci. Rep. 2, 319; DOI:10.1038/srep00319 (2012) 